\title{
Antifungal Activity of Selenium Nanoparticles Extracted from Capparis decidua Fruit against Candida albicans
}

\author{
Shifa Jawahar Ali1 ${ }^{1}$ Preetha S. ${ }^{2}$, Jeevitha $\mathrm{M}^{3}$ Lavanya Prathap ${ }^{4}$, Rajeshkumar S. ${ }^{5}$
}

\begin{abstract}
${ }^{1}$ Department of Physiology, Saveetha Dental College and Hospitals, Saveetha Institute of Medical and Technical Sciences (SIMATS), Chennai, Tamilnadu, India. ${ }^{2}$ Department of Physiology, Saveetha Dental College and Hospitals, Saveetha Institute of Medical and Technical Sciences (SIMATS), Chennai, Tamilnadu, India. ${ }^{3}$ Department of Periodontics, Saveetha Dental College and Hospitals, Saveetha Institute of Medical and Technical Sciences (SIMATS), Chennai, Tamilnadu, India. ${ }^{4}$ Department of Anatomy, Saveetha Dental College and Hospitals, Saveetha Institute of Medical and Technical Sciences

(SIMATS), Chennai, Tamilnadu, India. ${ }^{5}$ Department of Pharmacology, Saveetha Dental College and Hospitals, Saveetha Institute of Medical and Technical Sciences (SIMATS), Chennai, Tamilnadu, India.
\end{abstract}

\section{ABSTRACT}

\section{BACKGROUND}

Nanotechnology has introduced nanoparticulate form of selenium for a wide variety of applications. Nanoparticles can be used in medicine due to their increased interaction with microbes and also because it has fewer side effects than the drugs. Selenium nanoparticles have unique biomedical applications ranging from antioxidant activity to anticancer activity. It is distinct with its high biological activity and low toxicity and cytotoxic property. Capparis decidua also called as karira belonging to Capparaceae family is a xerophytic small branched shrub found in Africa, Middle East and South Asia. Its fruit is used for preparing curry and pickles in the western parts of India. In our study, the selenium nanoparticles were extracted from its fruit. Candida albicans is pathogenic yeast in the oral cavity, gastrointestinal tract and the urogenital tract affecting the immunocompromised patients and causes various types of infections. It has gained resistance to present antifungals and new formulations are needed to be discovered.

\section{METHODS}

Plant extract was prepared from Capparis decidua fruit powder and filtered using Whatman No. 1 filter paper. It was kept in magnetic stirrer for nanoparticle synthesis. Colour change was observed which indicates the synthesis of nanoparticles. UV visible spectroscopy was taken in proper intervals. The nanoparticles synthesized were purified by centrifugation technique and checked for its antifungal activity. Antifungal activity of the prepared nanoparticles against Candida albicans was determined by using agar well diffusion assay method or agar disc diffusion method. The zone of inhibition formed around the disc indicates the sensitivity of the fungi to the plant extract. The inhibition zone diameter was measured using a ruler and compared with the inhibition zone formed by the positive control drug which was done in parallel.

\section{RESULTS}

Antifungal activity was showed by an inhibition zone which was characterized by a clear zone around the wells. The diameter of the inhibition zone formed for $50 \mathrm{~mL}$ of the plant extract was $20.33 \mathrm{~mm} \pm 0.47$, for $100 \mathrm{~mL}$ of the plant extract was 28.33 $\mathrm{mm} \pm 0.47$, for $150 \mathrm{~mL}$ of the plant extract was $30.33 \mathrm{~mm} \pm 0.47$ and for the positive control drug was $34.33 \mathrm{~mm} \pm 0.47$.

\section{CONCLUSIONS}

The diameter of the inhibition zones was compared with the positive control drug. Selenium nanoparticles extracted from Capparis decidua fruit showed high activity against Candida albicans. Further in vivo research can be done for the same and it may represent an alternative for treating fungal infections.

\section{KEY WORDS}

Selenium Nanoparticles, Capparis decidua, Antifungal Activity, Candida albicans, Fungi, Plant Extract
Corresponding Author:

Dr. Preetha S.,

Department of Physiology

Saveetha Dental College and Hospitals,

Saveetha Institute of Medical and

Technical Sciences (SIMATS),

Saveetha University, 162,

Poonamallee High Road

Chennai- 600077, Tamilnadu, India.

E-mail:drpreeth.homeo@gmail.com

DOI: $10.14260 /$ jemds/2020/533

How to Cite This Article:

Ali SJ, Preetha S, Jeevitha M. et al. Antifungal activity of selenium nanoparticles extracted from capparis decidua fruit against candida albicans. Evolution Med Dent Sci 2020;9(34):24522455, DOI: $10.14260 /$ jemds/2020/533

Submission 09-05-2020,

Peer Review 10-07-2020,

Acceptance 17-07-2020,

Published 24-08-2020.

Copyright (c) 2020 JEMDS. This is an open access article distributed under Creative Commons Attribution License [Attribution 4.0 International (CC BY 4.0)] 


\section{BACKGROUND}

Nanoparticles are the particles of size ranging between 1 to $100 \mathrm{~nm}$ in diameter and occur in different shapes. They occur in nature extensively and they are studied widely in science. They can easily pass through the common filters due to their extremely smaller size and also a cause of atmospheric pollution. They cannot be seen in normal microscopes but can be only seen in electron microscopes. They are used in almost all fields of science and also have many applications in textile industry, cosmetics, electronics and also food industry. The use of nanoparticles has increased drastically in the recent years and will keep increasing in future due to the advanced researches going on. Nanotechnology is believed to be the next industrial revolution as it can have enormous impact on present society and in future. [1] The standard methods of synthesizing nanoparticles have many limitations like the cost, generation of toxic chemicals.[2] Nanoparticles have greater surface area than microparticles which leads to increased interactions with the microbial cells. ${ }^{[3]}$ Hence when used in medicine, it is safe and has less side effects by reducing damage to healthy cells. Wavelength of nanoparticles lies below the critical wavelength of light which causes them to be transparent. This property contributes to their wide applications in cosmetics, coatings and packaging. [4]

Selenium is a chemical element with atomic number 34. It is a non-metal belonging to the $16^{\text {th }}$ group and have six stable isotopes called nuclear isomers.[1] It is found as pure compound or in its elemental state in the earth's crust. It is present in humans in less amounts and is toxic if present in more concentration. It is used as an additive to glass. Selenium is also used to reduce the transmission of sunlight by giving a bronze tint to the architectural glass. It is widely used in rectifiers due to its ability to convert AC electricity to DC electricity. It is also used as an additive for making stainless steel and is used in vulcanization of rubber to increase its resistance to abrasion. It acts as an environmental pollutant in higher concentration. Selenium is an essential micronutrient for humans and animals because it is involved in the synthesis of main selenoproteins such as glutathione peroxide and thioredoxin reductase. Selenium exists in three allotropic forms such as the red coloured amorphous selenium, the black coloured crystalline trigonal and the red coloured crystalline monoclinic.[5] Black crystalline form can be used in photocells and it conducts electricity in the light better than the dark.

Selenium nanoparticles has unique biomedical applications ranging from antioxidant activity, antibacterial, antiviral to anticancer activity.[6] It exhibits therapeutic activity against different types of oxidative stress and inflammation driven disorders. Selenium nanoparticle is distinct with its high biological activity and low toxicity and cytotoxic property.[7],[8] It exhibits reduced toxicity compared to different selenium species. ${ }^{[9]}$ Selenium nanoparticles have also lower cytotoxic effects than silver nanoparticles because of these characteristics it is gaining wide attention among researchers.[10] Capparis decidua also called as karira belonging to Capparidaceae family is a xerophytic small branched shrub found in Africa, Middle East and South Asia. It is a drought resistant plant. It is widely used in folk medicine and its fruit is recommended for diabetic patients. Its spicy fruits are used for preparing curry and fine pickles in the western parts of India. Its fruits are rich in protein and minerals and also have a high fat content. The protein content and mineral constituents of Capparis decidua were found to be higher than the common fruits. The plant is also used traditionally for curing pain in tooth, asthma, inflammation, cough, arthritis, malaria and swellings. New plants can be cultivated easily from cuttings. Its wood is also used for many things.

Previously antifungal effect of silver nanoparticles was studied against Candida albicans and Selenium Nanoparticles Synthesized from various sources were studied for its properties and its activity against various fungi. Selenium sulphide is a selenium compound which have been used as an antifungal agent over a long time but the antifungal activity of Capparis decidua fruit extracted selenium nanoparticles has not yet been investigated against candida albicans.[11]

Candida albicans is pathogenic yeast in the oral cavity, gastrointestinal tract and the urogenital tract affecting the immunocompromised patients and causes various types of infections. ${ }^{[12]}$ Candida albicans can cause infections that can affect different parts of the body and is difficult to treat and some invasive infections can be life threatening. Recently Candida albicans has acquired resistance to the main antifungals. [13], [14] gradually all the Pathogenic fungi can gain resistant to the existing antifungal agents and hence development of new antifungal formulations is essential. In the present study selenium nanoparticles were extracted and purified from the Capparis decidua fruit and the antifungal activity of the prepared selenium nanoparticles were investigated by agar well diffusion assay method.

\section{METHODS}

$1 \mathrm{~g}$ of Capparis decidua fruit powder was mixed in $100 \mathrm{~mL}$ of water and kept in a water bath for 1 hour at $90^{\circ} \mathrm{C}$. After one hour, the extract was filtered using Whatman No. 1 filter paper and was kept in a separate beaker for further use.

$30 \mathrm{~mL}$ of the prepared plant extract was mixed with 70 $\mathrm{mL}$ of distilled water. $30 \mathrm{~mL}$ of sodium selenite was prepared with the $100 \mathrm{~mL}$ of the solution and kept in the magnetic stirrer for nanoparticle synthesis. Colour change was observed which indicated the synthesis of nanoparticles (figure 1). UV Visible spectroscopy reading was taken in correct intervals. The synthesized nanoparticles were purified by using centrifugation technique and checked for its antifungal activity against candida albicans.

Antifungal activity of the synthesized selenium nanoparticles was determined using the agar well diffusion assay method which is also called as agar disc diffusion method. The sensitivity of the fungi to the extract is determined by the formation of zone of inhibition around the disc. Stock culture of Candida albicans were prepared and maintained in saturated dextrose agar slants at $4^{\circ} \mathrm{C}$. The petri dish was incubated under suitable conditions. A positive control drug was also done parallel to compare the results. Generally, the antifungal agents diffuse into the agar and inhibits the growth of the microorganism forming a zone around them. The plates were examined for the evidence of zone of inhibition, which can be distinguished by the 
appearance of clear area around the wells. The diameter of such zones were measured using a ruler and noted down.

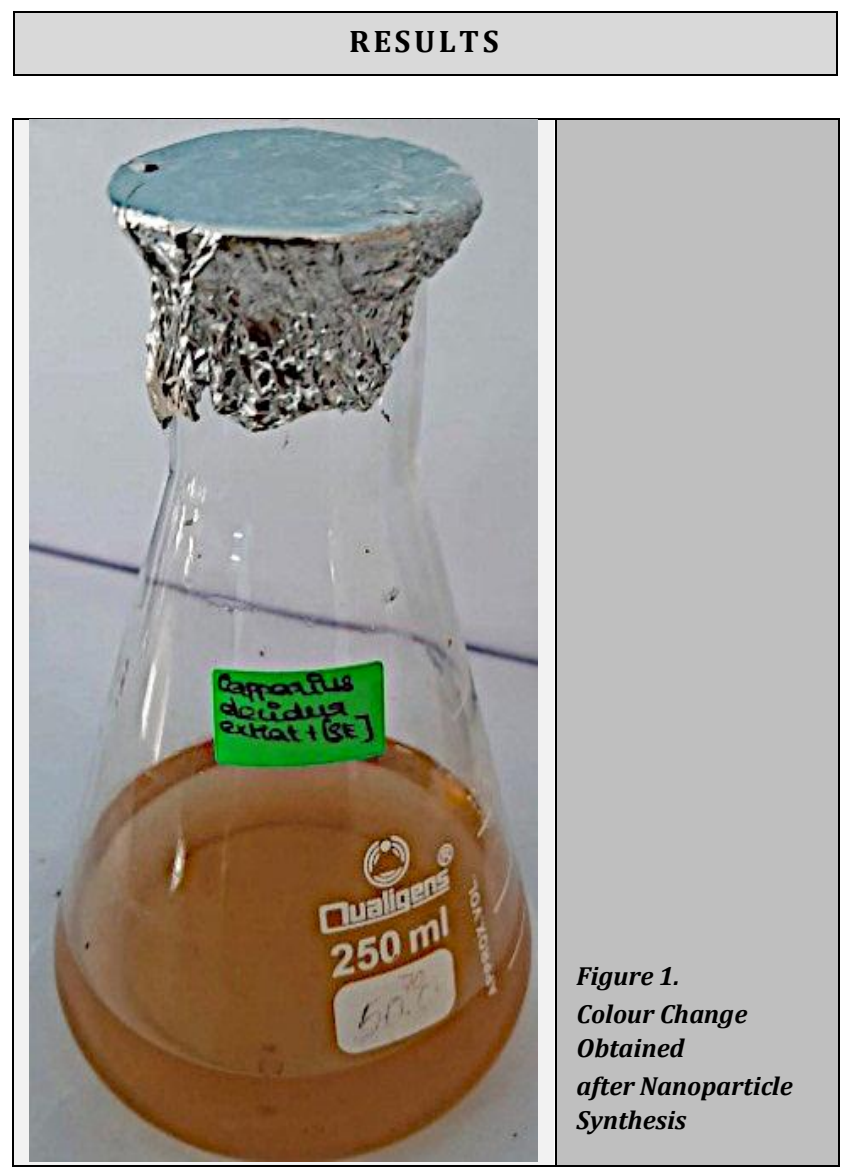

\begin{tabular}{|c|c|c|c|c|c|c|}
\hline $\begin{array}{c}\text { Concent } \\
\text {-ration } \\
\text { (mL) }\end{array}$ & $\begin{array}{r}\text { Zone } \\
\text { Reading } \\
1(\mathrm{~mm})\end{array}$ & $\begin{array}{l}\text { of Inhib } \\
\text { Reading } \\
2(\mathrm{~mm})\end{array}$ & $\begin{array}{l}\text { bition } \\
\text { geading } \\
3(\mathrm{~mm})\end{array}$ & Mean & S.D. & $\begin{array}{c}\text { Diameter of } \\
\text { Inhibition } \\
\text { Zone (mm) }\end{array}$ \\
\hline 50 & 20 & 21 & 20 & 20.33333333 & 0.471404521 & $20.33 \pm 0.47$ \\
\hline 100 & 28 & 28 & 29 & 28.33333333 & 0.471404521 & $28.33 \pm 0.47$ \\
\hline 150 & 30 & 31 & 30 & 30.33333333 & 0.471404521 & $30.33 \pm 0.47$ \\
\hline $\mathrm{AB}$ & 34 & 34 & 35 & 34.33333333 & 0.471404521 & $34.33 \pm 0.47$ \\
\hline
\end{tabular}

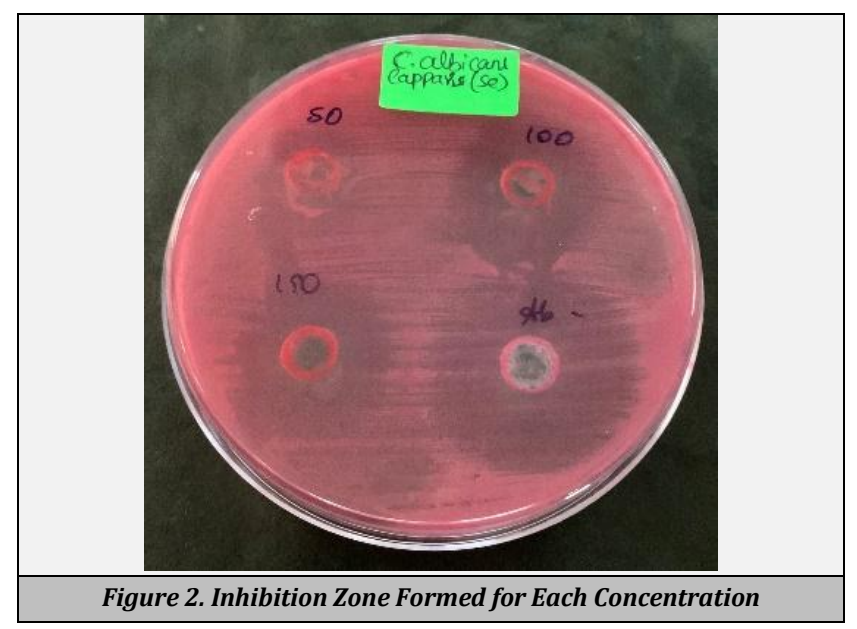

Selenium nanoparticles were successfully synthesized from Capparis decidua fruit powder which was evident by a significant colour change in the cultivation medium. Antifungal activity was showed by an inhibition zone which was characterized by a clear zone between the wells containing samples and a certain distance (figure 2). The diameter of inhibition zone formed for each concentration is tabulated below (table 1). A graph is done to analyse the difference in the diameter of the inhibition zone with each concentration of the pant extract and compared with the positive control drug done parallel to it. (Graph 1).

The diameter of the inhibition zone formed for $50 \mathrm{~mL}$ of the plant extract was $20.33 \mathrm{~mm} \pm 0.47$, for $100 \mathrm{~mL}$ of the plant extract was $28.33 \mathrm{~mm} \pm 0.47$, for $150 \mathrm{~mL}$ of the plant extract was $30.33 \mathrm{~mm} \pm 0.47$ and for the positive control drug was $34.33 \mathrm{~mm} \pm 0.47$

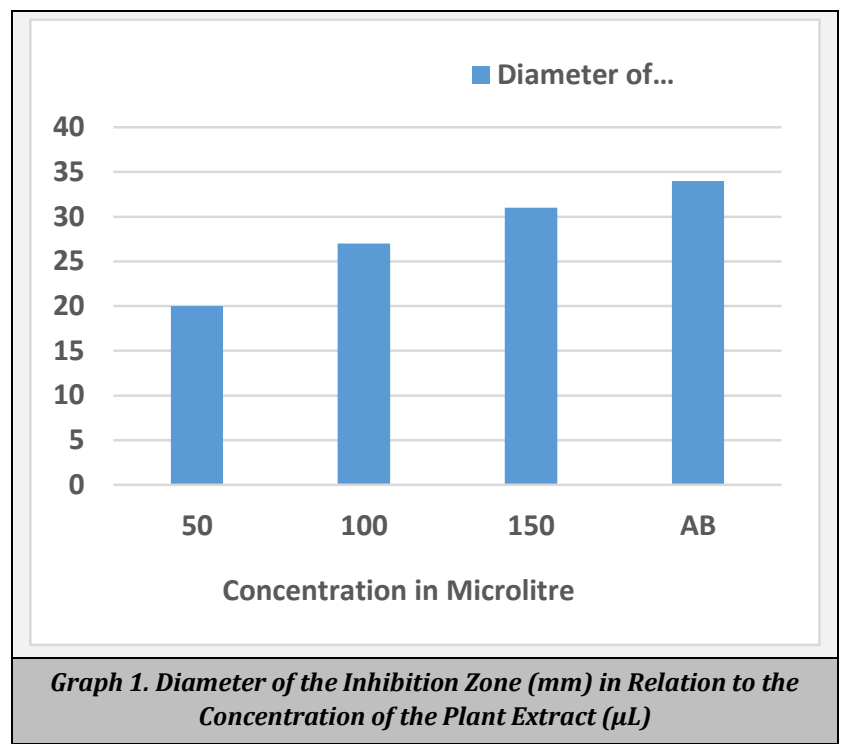

DISCUSSION

These results obtained shows that selenium nanoparticles obtained from Capparis decidua fruit has fungistatic and fungicidal properties against the microorganism tested. Hence it can be used as a potential candidate for developing new antimicrobial agents with a wide range of applications. [15] It could represent an alternative for treating fungal infections. Nanoparticles when used as antimicrobial agents have comparatively less side effects than the other antimicrobial drugs. They are also more effective due to their increased surface area which leads to increased interaction with the microbes.

A study done to find the antimicrobial effects of selenium nanoparticle-enriched probiotics and their fermented broth against Candida albicans, selenium nanoparticles showed good antifungal activity against Candida albicans which is similar to our results. A greater decrease in viability of Candida albicans were seen for bacteria grown in the presence of selenium dioxide than for the non-selenium enriched bacteria.

A study was done on selenium nanoparticles biosynthesized using bee propolis which is a resinous mixture produced by bees. The beehive product was collected from five different Indian states were used for the biosynthesis of selenium nanoparticles. The antioxidant and antimicrobial activities of the synthesized selenium nanoparticles were evaluated. The antifungal effect of selenium nanoparticles was evaluated by well diffusion 
method and the inhibition zone formed for Aspergillus Niger was $20 \mathrm{~mm}$ Aspergillus flavours was $18 \mathrm{~mm}$ but no significant effect was seen for Candida albicans. The result in this study is different from the result obtained in our present study and the majority of other studies. The variation in the result could be due to some differences in laboratory environment or the methods carried out.[16]

A study was done on selenium nanoparticles synthesized by Bacillus species MSh-1 and its antifungal activity was tested against Aspergillus fumigatus and Candida albicans. The results obtained from this study showed us that the biogenic selenium nanoparticles have good antifungal effect and are useful antifungal agents which are also similar to our results.

A Study was done on silver nanoparticles obtained by green synthesis against Candida albicans and Candida tropicalis. To avoid the toxic compounds produced during the synthesis process, silver nanoparticles were synthesized using ribose as a reducing agent and sodium dodecyl sulphate as a stabilizer. The antifungal activity was evaluated and was observed that silver nanoparticles showed high activity against Candida albicans and Candida tropicalis which could represent an alternative for fungal infection treatment.[15]

Another study done on biogenic selenium nanoparticles synthesized from electronic waste thereby making use of the electronic waste components and recycling them into nanoantifungal agent. The nanoparticles synthesized were analysed for its antifungal effect against Candida albicans and Aspergillus niger by disk diffusion method. When compared with both the fungi, selenium nanoparticles showed better activity against Candida albicans. ${ }^{[3]}$

\section{CONCLUSIONS}

In the present study Selenium nanoparticles extracted from Capparis decidua fruit was assessed for its antifungal activity and it showed high activity against the fungus Candida albicans. This should be further assessed for its practical application and could be used in treating diseases in medicine and healthcare. This property of selenium nanoparticles can be further improved and used in treating diseases in medicine and healthcare. Further in-vivo research can be done and may represent an alternative for treating fungal infections.

Financial or Other Competing Interests: None.

\section{REFERENCES}

[1] Sarkar J, Dey P, Saha S, et al. Mycosynthesis of selenium nanoparticles. Micro \& Nano Letters. IET 2011; 6(8):599602.

[2] Deepa B, Ganesan V. Biogenic synthesis and characterization of selenium nanoparticles using the flower of bougainvillea spectabilis willd. Int J Sci Res 2015; 4(1):690-5.
[3] Eswarapriya B, Jegatheesan KS. Antifungal activity of biogenic selenium nanoparticles synthesized from electronic waste. Int J Pharm Tech Res 2015; 8(3):383-6.

[4] Thakkar KN, Mhatre SS, Parikh RY. Biological synthesis of metallic nanoparticles. Nanomedicine 2009; 6(2):25762.

[5] Ismail AWA, Sidkey NM, \& Arafa RA, et al. Evaluation of in vitro antifungal activity of silver and selenium nanoparticles against alternaria solani caused early blight disease on potato. Br Biotechnol J 2016; 12(3):111.

[6] Tran PA, Webster TJ. Selenium nanoparticles inhibit staphylococcus aureus growth. Int J Nanomedicine 2011; 6:1553-8.

[7] Tammina SK, Mandal BK, Ranjan S, et al. Cytotoxicity study of Piper nigrum seed mediated synthesized $\mathrm{SnO} 2$ nanoparticles towards colorectal (HCT116) and lung cancer (A549) cell lines. J Photochem Photobiol B 2016; 166:158-68.

[8] Greeshma B, Mahesh M. Biosynthesis of selenium nanoparticles from Bacillus species and its applications. Journal of Applied and Natural Science 2019; 11(4):8105.

[9] Khurana A, Tekula S, Saifi M, et al. Therapeutic applications of selenium nanoparticles. Biomedicine \& Pharmacotherapy 2019; 111.802-12.

[10] Vahdati M, Moghadam TT. Synthesis and characterization of selenium nanoparticles-lysozyme nanohybrid system with synergistic antibacterial properties. Sci Rep 2020; 10:510.

[11] Shakibaie M, Mohazab NS, Mousavi SAA. Antifungal activity of selenium nanoparticles synthesized by bacillus species Msh-1 against aspergillus fumigatus and candida albicans. Jundishapur J Microbiol 2015; 8(9):e26381.

[12] Kheradmand E, Rafii F, Yazdi MH, et al. The antimicrobial effects of selenium nanoparticle-enriched probiotics and their fermented broth against Candida albicans. Daru 2014; 22(1):48.

[13] Wang T, Yang L, Zhang B, et al. Extracellular biosynthesis and transformation of selenium nanoparticles and application in $\mathrm{H} 2 \mathrm{O} 2$ biosensor. Colloids Surf B Biointerfaces 2010; 80(1):94-102.

[14] Mallmann, EJJ, Cunha FA, Castro BNMF, et al. Antifungal activity of silver nanoparticles obtained by green synthesis. Rev Inst Med Trop São Paulo 2015; 57(2):1657.

[15] Machado A, Wady AF, Zamperini CA, et al. Antifungal activity of silver nanoparticles against candida albicans. Journal of Nanomaterials 2013; 2013:1-9.

[16] Shubharani R, Mahesh M, Yogananda Murthy VN. Biosynthesis and characterization, antioxidant and antimicrobial activities of selenium nanoparticles from ethanol extract of bee propolis. J Nanomed Nanotechnol 2019; $10: 1$. 